\title{
Apparently normal epicardial coronaries in a patient with inferior wall myocardial infarction on the background of mild coronavirus disease-2019: take a second look!
}

\author{
Barun Kumar ${ }^{1}$, Ashwin Kodliwadmath ${ }^{1}$, Amar Upadhyay ${ }^{2}$, Anupam Singh ${ }^{3}$, Nanda $\mathbf{N}^{4}$ \\ ${ }^{1}$ Department of Cardiology, All India Institute of Medical Sciences (AIIMS), Rishikesh; ${ }^{2}$ Department of Pediatrics, \\ Doon Medical College, Dehradun; ${ }^{3}$ Department of Ophthalmology, All India Institute of Medical Sciences (AIIMS), \\ Rishikesh; ${ }^{4}$ Department of Endocrinology, All India Institute of Medical Sciences (AIIMS), Rishikesh, India \\ This study was carried out in Department of Cardiology, All India Institute of Medical Sciences (AIIMS), Rishikesh, \\ Uttarakhand, India
}

\begin{abstract}
The coronary angiographic (CAG) findings of ST elevation myocardial infarction (STEMI) in patients of coronavirus disease 2019 (COVID-19) range from increased coronary artery thrombus
\end{abstract}

Correspondence: Ashwin Kodliwadmath, MD, Department of Cardiology, All India Institute of Medical Sciences (AIIMS), Rishikesh 249203, Uttarakhand, India.

Tel. +91.8312470157.

E-mail: ashrocks33@gmail.com

Ethics approval and consent to participate: No ethical committee approval was required for this case report by the Department, because this article does not contain any studies with human participants or animals.

Consent for publication: The authors confirm that written consent for publication of this case report including images and associated text has been obtained from the patients' guardians in line with COPE guidance.

Availability of data and material: All data has been incorporated in the manuscript.

Competing interests: The authors declare that they have no competing interests, and all authors confirm accuracy.

Contributions: All the authors made a substantive intellectual contribution. All the authors have read and approved the final version of the manuscript and agreed to be accountable for all aspects of the work.

Keywords: COVID-19; ST elevation myocardial infarction; coronary angiography; thrombosis.

Received for publication: 7 November 2020.

Accepted for publication: 4 March 2021.

${ }^{\circ}$ Copyright: the Author(s), 2021

Licensee PAGEPress, Italy

Monaldi Archives for Chest Disease 2021; 91:1668

doi: $10.4081 /$ monaldi.2021.1668

This article is distributed under the terms of the Creative Commons Attribution Noncommercial License (by-nc 4.0) which permits any noncommercial use, distribution, and reproduction in any medium, provided the original author(s) and source are credited. burden to normal coronaries due to STEMI mimics. Here we report the case of a 45 -year-old gentleman who presented with evolved inferior wall myocardial infarction with ongoing angina along with mild COVID-19. CAG showed normal epicardial coronaries except for distal right posterior descending coronary artery (RPDA) $100 \%$ occlusion on careful examination. He was treated for the myocardial infarction with medical management along with treatment of COVID-19. The importance of our case is to highlight the possibility of distal total occlusion of small coronary branches which maybe missed if not carefully looked for as a normal CAG in COVID-19 patient will require only supportive therapy, while the finding of distal $100 \%$ occlusion of RPDA deemed us to prescribe optimal medical therapy as per acute myocardial infarction protocol along with treatment for COVID-19.

\section{Introduction}

Patients of coronavirus disease 2019 (COVID-19) are found to have a hypercoagulable state known as COVID-19 associated hemostatic abnormalities (CAHA). The prothrombotic state can include venous thromboembolism (VTE), stroke, acute limb ischemia, aortic thrombosis, splenic infarcts and acute coronary syndrome (ACS) [1]. Patients of COVID-19 who present with ST elevation myocardial infarction (STEMI) have variable coronary anatomy on coronary angiography $(\mathrm{CAG})$, with few reports of increased coronary artery thrombus burden [2], and few reports of STEMI with normal epicardial coronaries $[3,4]$.

\section{Case report}

Here, we report the case of a 45 -yea-old gentleman, type 2 diabetic, non-smoker who presented to us with a 3-day history of retrosternal chest pain radiating to left arm associated with sweating. He reported history of fever, dry cough and sore throat for 4 days but no history of loose stools, breathlessness or loss of smell or taste. Twelve lead surface electrocardiogram (ECG) showed q with ST elevation in leads III and aVF with no reciprocal changes (Figure 1A) with a peak troponin I of $20.5 \mathrm{mcg} / \mathrm{L}$ (normal $<0.05 \mathrm{mcg} / \mathrm{L}$ ), suggestive of evolved inferior wall myocardial infarction (IWMI). Transthoracic echocardiography showed 
hypokinesia in the right coronary artery (RCA) territory with left ventricular ejection fraction (LVEF) of $45 \%$ with normal right ventricular function. He was loaded with tablet aspirin $325 \mathrm{mg}$, tablet clopidogrel $600 \mathrm{mg}$ and tablet atorvastatin $80 \mathrm{mg}$. Reverse transcriptase polymerase chain reaction (RT-PCR) nasopharyngeal (NP) and oropharyngeal (OP) swab tested positive for SARS-CoV-2. His oxygen saturation on room air was $99 \%$. The investigation panel including complete blood count, renal function tests, liver function tests, serum ferritin, interleukin-6, lactate dehydrogenase and ddimer levels were within the normal range. High-resolution computed tomography thorax was within normal limits. In view of the ongoing angina, he was taken up for coronary angiography (CAG) from the right radial route. The left coronary system was normal (Figure 1 B-D). Right coronary system also appeared normal at the first look (Figure 1 E,F). Careful review of the RCA CAG showed abrupt cut-off of the distal right posterior descending coronary artery (RPDA), suggestive of distal RPDA 100\% occlusion (Figure 2 A,B; Videos 1 and 2). The patient was kept on medical management for the IWMI with injection enoxaparin $60 \mathrm{mg}$ subcutaneous twice daily for 5 days, dual antiplatelets, tablet aspirin $150 \mathrm{mg}$ once daily and tablet clopidogrel $75 \mathrm{mg}$ once daily, tablet atorvastatin 40 $\mathrm{mg}$ once daily, tablet metoprolol succinate $50 \mathrm{mg}$ once daily, tablet nitroglycerine $2.6 \mathrm{mg}$ twice daily along with oral hypoglycemic agents for diabetes. Treatment for COVID-19 included tablet vitamin C $500 \mathrm{mg}$ thrice daily, tablet zinc $50 \mathrm{mg}$ once daily, tablet paracetamol $500 \mathrm{mg}$ thrice daily and tablet favipiravir $1800 \mathrm{mg}$ twice on day 1 followed by $800 \mathrm{mg}$ twice daily for 7 days. $\mathrm{He}$ improved with RT-PCR NP abd OP swab for SARS-CoV-2 turning out negative on day 8 . He was advised a follow-up CAG to look for the RPDA distal total occlusion, however the patient refused.
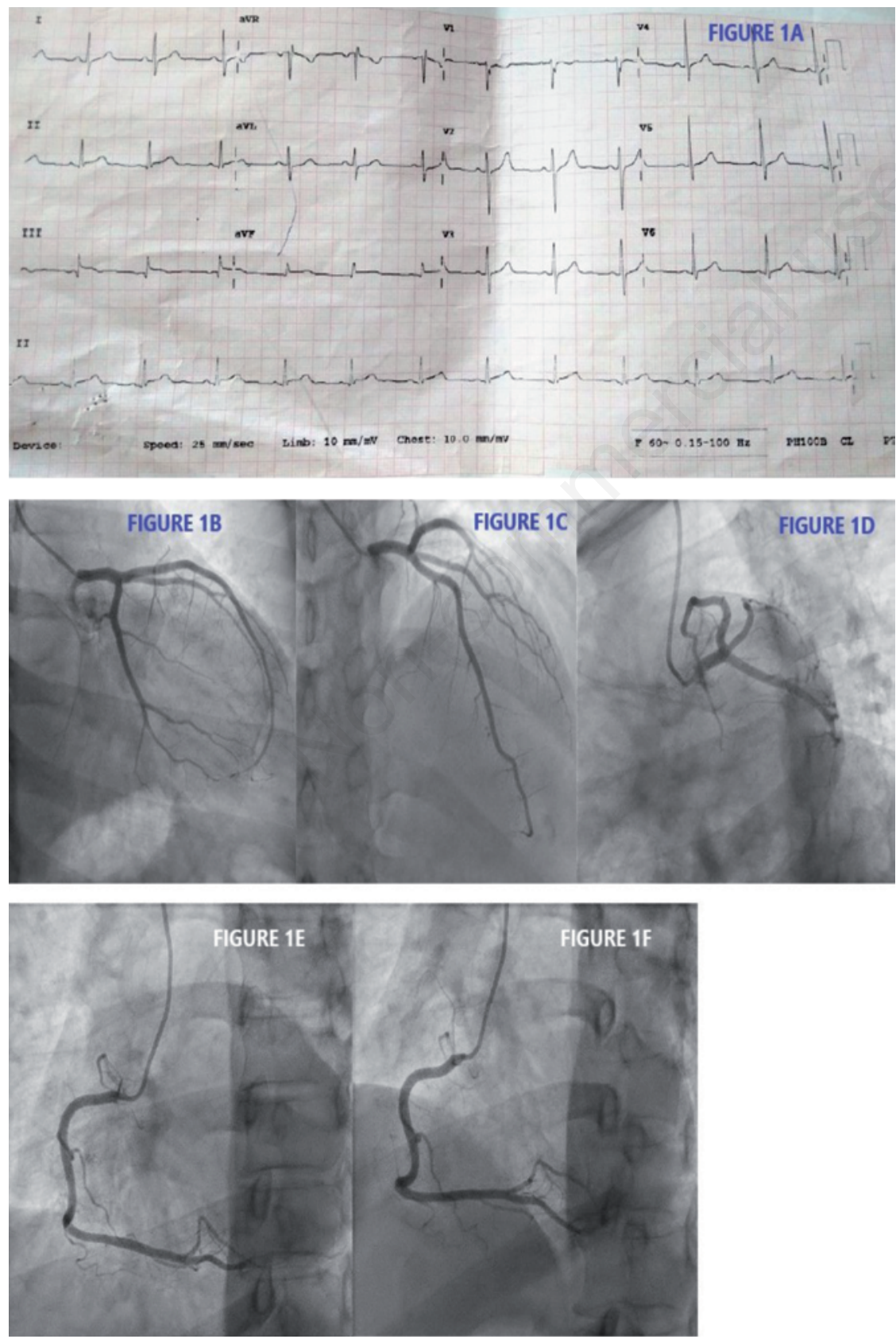

Figure 1. A) Twelve lead surface electrocardiogram showing sinus rhythm with $\mathrm{q}$ with ST elevation in leads III and aVF. B) Left coronary angiogram done through right radial route in right anterior oblique caudal view showing normal left main coronary artery, normal type III left anterior descending coronary artery, normal non-dominant left circumflex coronary artery and a normal ramus intermedius branch. C) Left coronary angiogram done through right radial route in antero-posterior cranial view showing normal left main coronary artery, normal type III left anterior descending coronary artery, normal left circumflex coronary artery and a normal ramus intermedius branch. D) Left coronary angiogram done through right radial route in left anterior oblique caudal view showing normal left main coronary artery, normal left anterior descending coronary artery, normal nondominant left circumflex coronary artery and a normal ramus intermedius branch. E) Right coronary angiogram done through right radial route in left anterior oblique $30^{\circ}$ caudal $5^{\circ}$ view showing dominant right coronary artery with normal proximal, mid and distal segments, with normal conus, right ventricular and acute marginal branches, terminating into posterior descending and postero-lateral branches. F) Right coronary angiogram done through right radial route in left anterior oblique $34^{\circ}$ cranial $12^{\circ}$ view showing dominant right coronary artery with normal proximal, mid and distal segments, with normal conus, right ventricular and acute marginal branches, terminating into posterior descending and postero-lateral branches. 


\section{Discussion}

Angiotensin-converting enzyme 2 (ACE2) has been identified as a functional receptor for coronaviruses, which is highly expressed in type II pneumocytes in the respiratory and cardiovascular systems resulting in cardiovascular manifestations of COVID-19. Patients with COVID-19 develop a hypercoagulable state known as CAHA [1]. Increased number of extramedullary megakaryocytes in vascular beds of lungs and hearts plays an important role in the increased thrombotic risk of COVID-19 patients $[5,6]$. In patients who require hospitalization, the rate of any thrombotic event is approximately $16 \%$, varying between $11.5 \%$ in non-intensive care unit (ICU) to $29.4 \%$ in ICU settings [7]. The CAHA is divided into three stages. Stage one includes patients with mild symptoms who show pulmonary microthrombi of peripheral microvasculature which may not be detected by computed tomography. Stage two includes patients who may develop severe symptoms requiring ICU support who show lung ventilation/perfusion impairment caused by thrombi or emboli on computed tomography scan and may have asymptomatic or symptomatic deep vein thrombosis (DVT). Stage three includes critically ill patients who need invasive mechanical ventilation or extracorporeal membrane oxygenation who exhibit VTE and extrapulmonary thrombosis involving several organs, such as intestine, limbs, and coronary or cerebral circulation [1]. Thus, coronary thrombosis occurs in CAHA stage 3. Patients may present with a clinical diagnosis of STEMI but have no evidence of obstructive epicardial coronaries $[1,3,4]$. ST elevation on the ECG in COVID-19 patients can occur due to myocarditis, microvascular thrombosis, cytokine-mediated injury, stressinduced cardiomyopathy, hypoxic injury, coronary spasm, and endothelial or vascular injury [8-10]. The prevalence of acute myocardial infarction (MI) in patients with COVID-19 ranges from $7-17 \%$ of hospitalized patients and over $20 \%$ of ICU patients [11].

The latest guidelines on management of acute MI during the COVID-19 pandemic, state that percutaneous coronary intervention (PCI) is the preferred therapy within $90 \mathrm{~min}$ from first medical contact, though fibrinolysis may be considered in patients who are "relatively stable" [11-13]. Patients with equivocal symptoms, atypical ECG, or delayed presentation and possible STEMI should undergo further evaluation including echocardiogram and serial ECGs [11].

As our patient had mild symptoms of COVID-19, he belonged to CAHA stage 1 and hence the acute IWMI and the COVID-19 were probably coincidental. The aim of our case report is not to draw conclusions on the relationship between the CAG findings and COVID-19. Small vessel occlusions can occur in otherwise any myocardial infarction patient with or without COVID-19. The background knowledge of the long list of differentials for STEMI mimics with normal epicardial coronaries among COVID-19 patients may lead clinicians to hastily arrive at a wrong diagnosis of normal coronaries and miss the important finding of distal total occlusion of side branches like RPDA as in our patient.

Hence our case report aims to highlight the possibility of acute distal total occlusion of branches like PDA or other side branches like septal, diagonal or marginal which can be missed if not specifically looked for, as a normal CAG in COVID-19 patient will require only supportive therapy, while the finding of $100 \%$ occlusion of distal RPDA deemed us to prescribe optimal medical therapy as per acute myocardial infarction protocol along with treatment for COVID-19.

\section{Conclusions}

Patients of COVID-19 have increased rates of thrombotic complications including acute STEMI. Patients of STEMI and COVID-19 can have normal epicardial coronaries due to various reasons. A careful review of the CAG images is mandated before labelling a COVID-19 patient with STEMI as having normal epicardial coronaries as distal total occlusion of small branches maybe missed on casual inspection of CAG images. A normal CAG would dictate supportive management for such patients, while acute total occlusions of small branches are the consequences of acute MI per se and require medical/interventional treatment for the same.

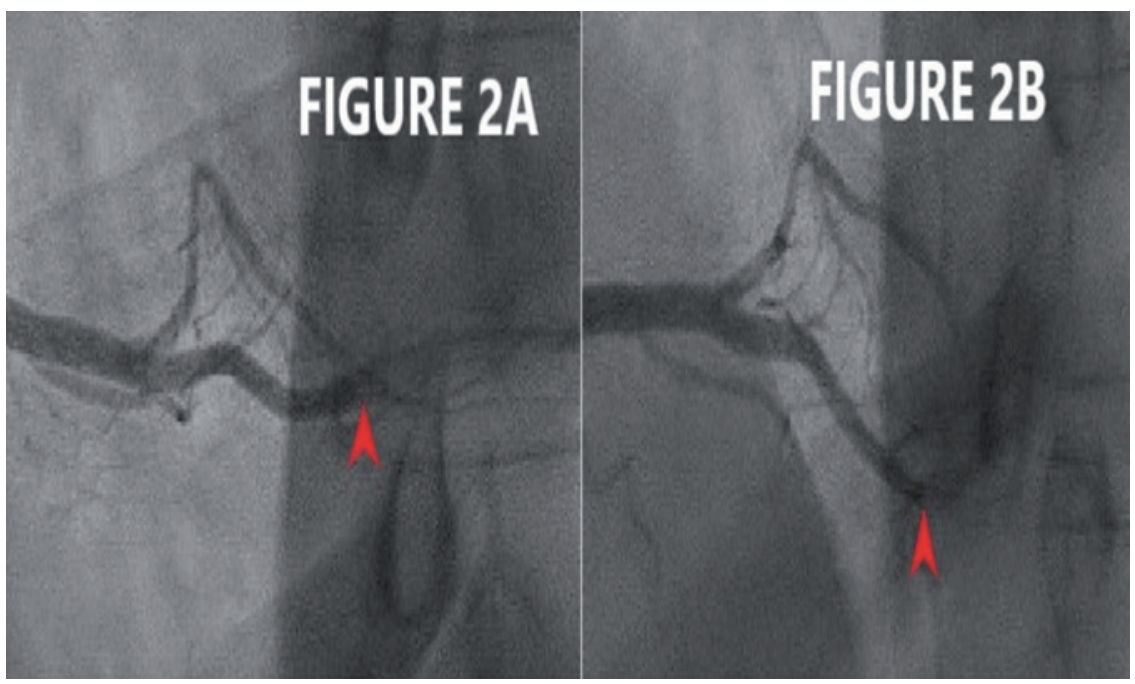

Figure 2. A) Right coronary angiogram done through right radial route in left anterior oblique $30^{\circ}$ caudal $5^{\circ}$ view, zoomed-in image showing abrupt cut-off of distal posterior descending coronary artery suggestive of total occlusion with normal posterolateral branches. B) Right coronary angiogram done through right radial route in left anterior oblique $34^{\circ}$ cranial $12^{\circ}$ view, zoomed-in image showing abrupt cut-off of distal posterior descending coronary artery suggestive of total occlusion with normal postero-lateral branches. 


\section{VIDEO LEGENDS}

Video 1. Fluoroscopic movie of right coronary angiogram done through right radial route in left anterior oblique $30^{\circ}$ caudal $5^{\circ}$ view showing abrupt cut-off of distal posterior descending coronary artery suggestive of total occlusion with normal postero-lateral branches.

Video 2. Fluoroscopic movie of right coronary angiogram done through right radial route in left anterior oblique $34^{\circ} \mathrm{cranial} 12^{\circ}$ view showing abrupt cut-off of distal posterior descending coronary artery suggestive of total occlusion with normal postero-lateral branches.

\section{References}

1. Ortega-Paz L, Capodanno D, Montalescot G, Angiolillo DJ. Coronavirus disease 2019-associated thrombosis and coagulopathy: review of the pathophysiological characteristics and implications for antithrombotic management. J Am Heart Assoc 2021;10:e019650.

2. Roffi M, Guagliumi G, Ibanez B. The obstacle course of reperfusion for ST-segment-elevation myocardial infarction in the COVID-19 pandemic. Circulation 2020;141:1951-3.

3. Bennett CE, Anavekar NS, Gulati R, et al. ST-segment elevation, myocardial injury, and suspected or confirmed COVID19 patients: diagnostic and treatment uncertainties. Mayo Clin Proc 2020;95:1107-11.

4. Cizgici AY, Agus HZ, Yildiz M. COVID-19 myopericarditis: it should be kept in mind in today's conditions. Am J Emerg Med 2020;38:1547.

5. Rapkiewicz AV, Mai X, Carsons SE, et al. Megakaryocytes and platelet-fibrin thrombi characterize multi-organ thrombosis at autopsy in COVID-19: a case series. EClinicalMedicine 2020;24:100434.

6 . Becker RC. COVID-19 update: COVID-19-associated coagulopathy. J Thromb Thrombolysis 2020;50:54-67.

7. Bilaloglu S, Aphinyanaphongs Y, Jones S, et al. Thrombosis in hospitalized patients with COVID-19 in a New York City health system. JAMA 2020;324:799-801.

8. Vidivich MI, Fischman DL, Bates ER. COVID-19 STEMI 2020 it's not what you know, it's how you think. JACC Case Rep 2020 27;2:1289-90.

9. Tavazzi G, Pellegrini C, Maurelli M, et al. Myocardial localization of coronavirus in COVID-19 cardiogenic shock. Eur J Heart Fail 2020;22:911-5.

10. Guzik TJ, Mohiddin SA, Dimarco A, et al. COVID-19 and the cardiovascular system: implications for risk assessment, diagnosis, and treatment options. Cardiovasc Res 2020;116:1666-87.

11. Avila J, Long B, Holladay D, Gottlieb M. Thrombotic complications of COVID-19. Am J Emerg Med 2021;39:213-8.

12. Welt FGP, Shah PB, Aronow HD, et al. Catheterization laboratory considerations during the coronavirus (COVID-19) pandemic: from the ACC's interventional council and SCAI. J Am Coll Cardiol 2020;75:2372-5.

13. Mahmud E, Dauerman HL, Welt FGP, et al. Management of acute myocardial infarction during the COVID-19 pandemic: a position statement from the society for cardiovascular angiography and interventions (SCAI), the American college of cardiology (ACC), and the American college of emergency physicians (ACEP). J Am Coll Cardiol 2020;76:1375-84. 